\title{
BEREZIN-TOEPLITZ QUANTIZATION OF THE MODULI SPACE OF FLAT $S U(N)$ CONNECTIONS
}

\author{
MARTIN SCHLICHENMAIER
}

Communicated by Theodore Voronov

\begin{abstract}
The moduli space of flat $\mathrm{SU}(n)$ connections on Riemann surfaces is of fundamental importance in TQFT. There is an associated representation of the mapping class group on the space of covariantly constant sections of the Verlinde bundle with respect to the AdPW-H connection. J. Andersen showed that this representation is asymptotically faithful. In his proof the Berezin-Toeplitz quantization of compact Kähler manifolds is used. In this contribution the background and some ideas of Andersen's proof is sketched.
\end{abstract}

\section{Introduction}

This is a write-up of a talk presented at the $\mathrm{XXV}^{\text {th }}$ Białowieża Workshop on Geometric Methods in Physics.

First, we recall the basics of the Berezin-Toeplitz quantization (operator and formal deformation quantization). Then we discuss the moduli space of flat $\operatorname{SU}(n)$ connections on a fixed Riemann surface in its different guises. Finally, we present recent results obtained by Andersen [3] showing the asymptotic faithfulness of the representations of the mapping class group (MCG, Teichmüller group) on the covariantly constant sections of the projectivized Verlinde bundle. In his approach he uses the Toeplitz operators and results on their correct semiclassical behavior as they will be presented in the first part.

As far as the Berezin-Toeplitz quantization is concerned the results were obtained by the author partly in joint works with Meinrenken, and Bordemann respectively with Karabegov [4], [10], [11], [12], [9].

Quite a number of mathematician (and physicists) were involved in the study of the moduli space of connections and the mapping class group. Instead giving references here, let me refer to the recent overviews by Jeffrey [6] and Masbaum [7]. The beautiful results on the asymptotic faithfulness presented are entirely due to Andersen [1]. For similar results in the $U(1)$ case, obtained by him, see [2]. 


\section{Berezin-Toeplitz Quantization of Compact Kähler Manifolds}

\subsection{Kähler Manifolds}

Let $(M, \omega)$ be a Kähler manifold, this says $M$ is a complex manifold, and $\omega$ a Kähler form, i.e., a closed (1,1)-form on $M$ which is positive.

The following manifolds are Kähler manifolds

1. The flat complex space $\mathbb{C}^{n}$ with the Kähler form $\omega=\mathrm{i} \sum_{i=1}^{n} \mathrm{~d} z_{i} \wedge \mathrm{d} \bar{z}_{i}$.

2. The complex projective line $\mathbb{P}^{1}(\mathbb{C})$, respectively the Riemann sphere with the form $\omega=\frac{\mathrm{i}}{(1+z \bar{z})^{2}} \mathrm{~d} z \wedge \mathrm{d} \bar{z}$, and more generally $\mathbb{P}^{n}(\mathbb{C})$ with the FubiniStudy form.

3. Every Riemann surface carries a Kähler form, and hence is a Kähler manifold.

4. Every (complex) torus of arbitrary dimension with the standard Kähler form on $\mathbb{C}^{n}$ introduced above.

5. Every (quasi-)projective manifold, i.e., every non-empty open subset of a projective variety without singularities, with the restriction of the FubiniStudy Kähler form of the embedding projective space.

6. Very often moduli spaces in the algebraic or analytic context carry a natural Kähler structure coming from their construction.

\subsection{Quantizable Kähler Manifolds}

Definition 1. (Quantization condition) A Kähler manifold $(M, \omega)$ is called quantizable, if there exists an associated quantum line bundle $(L, h, \nabla)$, i.e., a holomorphic line bundle $L$ over $M$, with hermitian metric $h$ on $L$, and compatible connection $\nabla$, fulfilling

$$
\operatorname{curv}_{\mathrm{L}, \nabla}=-\mathrm{i} \omega \text {. }
$$

By the compatibility requirements the connection will be uniquely fixed by the holomorphic structure and the metric on $L$.

Note that not all Kähler manifolds are quantizable. For example only those complex tori are quantizable which have enough theta functions, i.e., those which can 
be embedded holomorphically into projective space. They are called abelian varieties.

For the rest of this write-up we assume that $M$ is a compact Kähler manifold. We fix a quantum line bundle $L$ and consider its tensor powers $L^{m}:=L^{\otimes m}$, with metric $h^{(m)}$, and take $\Gamma_{\infty}\left(M, L^{m}\right)$ the space of smooth global sections, and $\Gamma_{h o l}\left(M, L^{m}\right)=\mathrm{H}^{0}\left(M, L^{m}\right)$ the subspace of global holomorphic sections.

Due to the compactness of $M$, the latter is finite-dimensional. On these spaces a scalar product is defined via

$$
\langle\varphi, \psi\rangle:=\int_{M} h^{(m)}(\varphi, \psi) \Omega, \quad \Omega:=\frac{1}{n !} \underbrace{\omega \wedge \omega \wedge \cdots \wedge \omega}_{n} .
$$

Let $\mathrm{L}^{2}(M, L)$ be the $\mathrm{L}^{2}$-completion and denote the projector to the closed subspace of holomorphic sections by

$$
\Pi^{(m)}: \mathrm{L}^{2}\left(M, L^{m}\right) \longrightarrow \Gamma_{h o l}\left(M, L^{m}\right) .
$$

\subsection{Berezin-Toeplitz Operator Quantization}

Fix $f \in C^{\infty}(M)$, and let $s \in \Gamma_{\text {hol }}\left(M, L^{m}\right)$ then the map

$$
s \quad \mapsto \quad T_{f}^{(m)}(s):=\Pi^{(m)}(f \cdot s)
$$

defines the Toeplitz operator of level $m$

$$
T_{f}^{(m)}: \quad \Gamma_{h o l}\left(M, L^{m}\right) \rightarrow \Gamma_{h o l}\left(M, L^{m}\right) .
$$

The Berezin-Toeplitz (BT) operator quantization is the map

$$
f \mapsto\left(T_{f}^{(m)}\right)_{m \in \mathbb{N}_{0}} .
$$

The reason to call it a quantization is, that it has the correct semi-classical behavior as expressed in the following theorem.

Theorem 2. (Bordemann, Meinrenken, and Schlichenmaier (BMS) [4])

$$
\begin{aligned}
\lim _{m \rightarrow \infty}\left\|T_{f}^{(m)}\right\| & =|f|_{\infty} \\
\left\|m \mathrm{i}\left[T_{f}^{(m)}, T_{g}^{(m)}\right]-T_{\{f, g\}}^{(m)}\right\| & =O(1 / m) \\
\left\|T_{f}^{(m)} T_{g}^{(m)}-T_{f \cdot g}^{(m)}\right\| & =O(1 / m) .
\end{aligned}
$$


In (2) the Poisson bracket for $C^{\infty}(M)$ is defined (using the Kähler form $\omega$ ) by

$$
\{f, g\}:=\omega\left(X_{f}, X_{g}\right), \quad \omega\left(X_{f}, .\right)=\mathrm{d} f(.)
$$

where $X_{f}$ is the Hamiltonian vector field associated to $f \in C^{\infty}(M)$.

The proofs of (2) and (3) are based on the symbol calculus of the generalized Toeplitz operators developed by Boutet de Monvel and Guillemin [5].

\subsection{Deformation Quantization}

Theorem 3. ([4], [9-12]). There exists a unique differential star product with the BT star product,

$$
f \star_{B T} g=\sum_{k=0}^{\infty} \nu^{k} C_{k}(f, g)
$$

such that

$$
T_{f}^{(m)} T_{g}^{(m)} \sim \sum_{k=0}^{\infty}\left(\frac{1}{m}\right)^{k} T_{C_{k}(f, g)}^{(m)}, \quad m \rightarrow \infty .
$$

This star product is of "separation of variables" type, and has classifying DeligneFedosov class $\frac{1}{\mathrm{i}}\left(\frac{1}{\nu}[\omega]-\frac{\epsilon}{2}\right)$, and corresponds to the Karabegov form $\frac{-1}{\nu} \omega+\omega_{\text {can }}$.

As usual the series in (4) has to be considered as a formal power series A star product is a differential star product if the $C_{k}(.,$.$) are bidifferential operators in their$ function arguments. Such a differential star product is of "separation of variables type" if the first function argument is only differentiated in holomorphic directions and the second argument only in anti-holomorphic directions (respectively the opposite directions depending on the convention chosen). This notion is due to Karabegov [8], and corresponds to the fact that the star product respects the complex structure. Such star products are classified by their formal Karabegov form. Above $\nu$ is used as formal variable for the forms and the formal forms are formal power series in $\nu$ if we ignore $1 / \nu$ which comes with the fixed $\omega$. In particular, for the BT star product no higher formal powers of $\nu$ occur in its classifying form. The Deligne-Fedosov class is a formal $H_{\text {deRahm }}^{2}(M)$ class which classifies the star product up to equivalence. The form $\omega_{\text {can }}$ is the curvature form of the canonical (holomorphic) line bundle with fibre metric coming from the Liouville form.

Note also that the asymptotic formula (5) is a short-hand notation for a very precise and strong asymptotic behaviour of the norms of the involved operators. See the cited references for the precise statement. 


\section{The Moduli Space of Flat SU $(n)$ Connections}

\subsection{Its Symplectic Structure}

Let $X$ be an oriented compact surface, and $p \in X$ a fixed point. We fix for the center of $\mathrm{SU}(n)$ a generator and then identify the center with $\mathbb{Z} / n \mathbb{Z}$. Let $\mathcal{A}_{F, \xi}$ be the set of flat $\mathrm{SU}(n)$ connections over $X \backslash\{p\}$ with holonomy $d \in \mathbb{Z} / n \mathbb{Z}$ around $p$. We denote by $G$ the group $\operatorname{SU}(n)$

The group of maps $X \rightarrow G$ from the surface $X$ to the group $G$ with point-wise multiplication in $G$, is the gauge group $\mathcal{G}$. It acts on the connection via gauge transformations

$$
A^{g}:=g^{-1} \mathrm{~d} g+g^{-1} A g .
$$

The moduli space of connections is the quotient of the set of connections modulo these gauge transformations

$$
\mathcal{M}:=\mathcal{A}_{F, \xi} / \mathcal{G} \cong \operatorname{Hom}_{d}\left(\pi_{1}(X \backslash\{p\}), G\right) / G
$$

The latter equivalence is the fact that this moduli space can be identified with the space of those group homomorphisms of the fundamental group $\pi_{1}(X \backslash\{p\})$ for which the basic loop around $p$ is mapped to $d \bmod n$ in the center of $G$, where the homomorphism are identified modulo conjugation in $G$.

Let $\mathcal{M}_{s}$ be the moduli space of irreducible flat connections (this corresponds to irreducible representations). It is a manifold, carries a natural symplectic structure $\omega$, and an associated hermitian line bundle $\mathcal{L}$ which is a quantum line bundle with respect to the symplectic structure. It is constructed from the WZW cocycle of the Chern-Simons action. See the appendix for more details and [6] for references and further information.

\subsection{Its Complex Structure}

We choose a complex structure $\sigma$ on $X$. This structure will induce complex structures on all introduced objects.

1. $X \Longrightarrow X^{\sigma}$ is now a (compact) Riemann surface

2. $\left(\mathcal{M}_{s}, \omega\right) \Longrightarrow\left(\mathcal{M}_{s}^{\sigma}, \omega^{\sigma}\right)$ is now a Kähler manifold

3. $\mathcal{L} \Longrightarrow \mathcal{L}^{\sigma}$ becomes a hermitian holomorphic line bundle, in fact, it is a quantum line bundle with respect to $\omega^{\sigma}$. 
Hence, $\mathcal{M}_{s}^{\sigma}$ is a quantizable Kähler manifold with quantum line bundle $\mathcal{L}^{\sigma}$. But what is the geometry of $\mathcal{M}_{s}^{\sigma}$ ? Is it compact? To study these questions we discuss another description of the moduli space.

\section{Holomorphic Rank $n$ Bundles Over Smooth Projective Curves}

\subsection{The Moduli Space}

Recall that the compact Riemann surface $X^{\sigma}$ can be identified with a smooth projective curve $C$ over $\mathbb{C}$. In the following we consider holomorphic vector bundles over $C$. First we define for every rank $n$ holomorphic vector bundle $E$, its determinant line bundle as $\operatorname{det} E:=\bigwedge^{n} E$, and its degree as $\operatorname{deg}(E):=\operatorname{deg}(\operatorname{det} E)$. The question is: Does there exist a moduli space of isomorphy classes of such bundles? The answer is: In generally not! We need to restrict our considerations to the subset of isomorphy classes of (Mumford) stable bundles, respectively S-equivalence classes of semi-stable bundles. A bundle $E$ is stable (respectively semi-stable) iff for every non-trivial subbundle $F$ of $E$ one has $\operatorname{deg}(F) / \mathrm{rk}(F)<\operatorname{deg}(E) / \mathrm{rk}(E)$ (respectively $\leq$ ). For the S-equivalence relation two semi-stable (but not stable) bundles are identified if certain associated graded objects are isomorphic.

Let $T$ be a line bundle and $n \in \mathbb{N}$. We use the following notations for the moduli spaces of bundles

$$
\begin{array}{llll}
U_{s}(n, d), & \operatorname{rk}(E)=n, & \operatorname{deg}(E)=d, & E \text { stable } \\
U_{s}(n, T), & \operatorname{rk}(E)=n, & \operatorname{det}(E)=T, & E \text { stable } \\
U(n, d), & \operatorname{rk}(E)=n, & \operatorname{deg}(E)=d, & E \text { semi-stable } \\
U(n, T), & \operatorname{rk}(E)=n, & \operatorname{det}(E)=T, & E \text { semi-stable. }
\end{array}
$$

In the following let $[p]$ be the line bundle corresponding to the divisor $p$, i.e., the line bundle which has a non-trivial section with exactly a zero of order one at $p$ and which is non-vanishing elsewhere. Furthermore let $d[p]$ be its $d$-tensor power. In particular, $\operatorname{deg} d[p]=d$.

We have the following properties:

1. $M:=U(n, d[p])$ is always projective algebraic (hence compact).

2. $M_{s}:=U_{s}(n, d[p])$ is Zariski open and smooth in $M$, hence a smooth manifold.

3. If $\operatorname{gcd}(n, d)=1$ then $M=M_{s}$, and hence $M_{s}$ is a compact Kähler manifold. 
4. The singularities of $M$ are rather mild.

5. For the Picard group Pic of isomorphy classes of line bundles we have $\operatorname{Pic}\left(M_{s}\right)=\operatorname{Pic}(M)=\mathbb{Z} \cdot[L]$, where $L$ is a special positive (respectively ample) line bundle

6. If we restrict $L$ to $M_{s}$ then $L \cong \mathcal{L}^{\sigma}$ introduced above. Furthermore, $\Gamma_{h o l}\left(M_{s}, L_{\mid}^{m}\right)=\Gamma_{h o l}\left(M, L^{m}\right)$.

7. If $g=2$ and $n=2$ then $M$ is always smooth.

The fundamental result is

$$
\mathcal{M}_{s}^{\sigma} \cong U_{s}(n, d[p])=M_{s}
$$

as complex manifold and as Kähler manifolds, and

$$
\mathcal{L}^{\sigma} \cong L
$$

as holomorphic line bundles.

A few names of people involved in showing these claims are Narasimhan, Seshadri, Weil, Mumford, Drezet, Ramadas, ...

\subsection{The Verlinde Bundle}

The Verlinde spaces are the vector spaces $\mathrm{H}^{0}\left(M, L^{m}\right)=\Gamma_{h o l}\left(M, L^{m}\right)$ and the dimension formula (as function of $m$ ) is called the Verlinde formula.

These Verlinde spaces are the quantum spaces, and the Berezin-Toeplitz operators

$$
T_{f}^{(m)}: \mathrm{H}^{0}\left(M, L^{m}\right) \rightarrow \mathrm{H}^{0}\left(M, L^{m}\right)
$$

are the quantum operators. We can apply Theorem 2 (BMS) and use the natural deformation quantization $\star_{B T}$ of Theorem 3. This works without modification if $M=M_{s}$, respectively if $M$ is smooth (e.g., if $\operatorname{gcd}(n, d)=1$ ). Otherwise the singularities need a special treatment, see Andersen [3].

We have to go one step further. If we consider the following diagram of associated objects we see that the first line does not depend on the complex structure $\sigma$, but the second does.

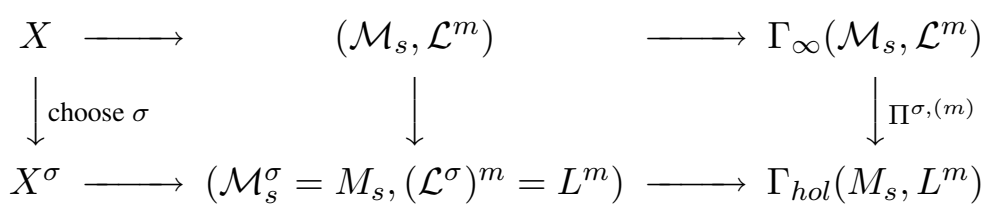


If we vary our $\sigma$ over the Teichmüller space $\mathcal{T}$, (i.e., the space of all complex structures on $X$ ) the first line will give trivial families of objects, the second line nontrivial families of objects over $\mathcal{T}$.

In particular, over $\mathcal{T}$ there is the trivial (infinite dimensional) bundle with fibre $\Gamma_{\infty}\left(\mathcal{M}_{s}, \mathcal{L}^{m}\right)$ which contains the subbundle $\mathcal{V}_{m}$ with fibre $\Gamma_{h o l}\left(M_{s}, L^{m}\right)$. The bundle $\mathcal{V}_{m}$ is called the Verlinde bundle over $\mathcal{T}$.

Fixing $f \in C^{\infty}\left(\mathcal{M}_{s}\right)$ its Toeplitz operator depends on the complex structure. Hence,

$$
\left(T_{f, \sigma}^{(m)}\right)_{\sigma \in \mathcal{T}}
$$

is a family of operators on the Verlinde bundle. In other words $T_{f,}^{(m)}$ is a section of $\operatorname{End}\left(\mathcal{V}_{m}\right)$.

\section{The Mapping Class Group (MCG) Action}

Over Teichmüller space $\mathcal{T}$ we have the bundles $\mathcal{V}_{m}$ and $\operatorname{End}\left(\mathcal{V}_{m}\right)$. We will discuss the following points:

1. There exists a naturally defined projectively flat connection $\nabla$ on $\mathcal{V}_{m}$, the Axelrod, della Pietra, Witten - Hitchin connection.

2. The MCG operates on the covariantly constant sections of $\mathbb{P}\left(\mathcal{V}_{m}\right)$.

3. Andersen [1] showed that this action of the MCG is asymptotically faithful (i.e., given an element $\gamma$ of the MCG, there is an $m$ such that $\gamma$ operates non-trivially).

Recall that the mapping class group(MCG) is defined as

$$
\Gamma:=\mathrm{MCG}:=\operatorname{Diff}^{+}(X) / \operatorname{Diff}_{0}(X)
$$

here $X$ is the surface of genus $g$, $\operatorname{Diff}^{+}(X)$ the group of orientation preserving diffeomorphisms and $\operatorname{Diff}_{0}(X)$ the subgroup of diffeomorphisms which are isotop to the identity.

First note that $\Gamma$ operates on our geometric objects as follows

1. By definition $\Gamma$ operates on the surface $X$

2. It operates on the Teichmüller space. In fact the moduli space $\mathcal{M}_{g}$ of isomorphism classes of compact genus $g$ Riemann surfaces (respectively smooth projective curves of genus $g$ ) is the quotient $\mathcal{T} / \Gamma$ 
3. It operates on $\operatorname{Hom}_{d}\left(\pi_{1}(X \backslash\{p\}), G\right) / G$

4. And hence it operates on $\mathcal{M}_{s}^{\sigma} \cong M_{s}$, the moduli spaces of irreducible connections, respectively stable bundles.

\subsection{Andersen's Result}

Let $\nabla$ be the Axelrod-della Pietra-Witten - Hitchin (AdPW-H) connection on $\mathcal{V}_{m}$ which is projectively flat. It induces a flat connection $\nabla^{\text {end }}$ on $\operatorname{End}\left(\mathcal{V}_{m}\right)$. We denote by $\mathbb{P}\left(W_{m}\right)$, the space of covariantly constant sections of $\mathbb{P}\left(\mathcal{V}_{m}\right)$ with respect to $\nabla$. Then the MCG operates also on $\mathbb{P}\left(W_{m}\right)$

$$
\rho_{m}: \Gamma \rightarrow \operatorname{Aut}\left(\mathbb{P}\left(W_{m}\right)\right) .
$$

Theorem 4. (Andersen, [1]) For $g \geq 3$ the map $\rho_{m}$ is asymptotically faithful. More precisely,

$$
\bigcap_{m=1}^{\infty} \operatorname{ker}\left(\rho_{m}\right)= \begin{cases}1, & g>2, \text { or } g=2, n>2, \text { or } \\ \{1, H\}, & g=2, n=2, \quad \text { odd } \\ \{=2, \quad \text { deven }\end{cases}
$$

where $H$ is the hyperelliptic involution, interchanging the branches of the $g=2$ hyperelliptic curve.

This says that in the generic case, given a non-trivial element of the mapping class group there exists a level $m$ such that the element operates non-trivially. This is the best to be hoped for. In the MCG the Dehn twists $\phi$ are elements of infinite order and it is known that the elements $\rho_{m}(\phi)$ have only finite order. Hence $\rho_{m}$ can never be faithful for finite $m$.

\subsection{Importance}

The assignment

$$
X \quad \longrightarrow \quad V(X)=\mathrm{H}^{0}\left(M_{s}, L^{m}\right)
$$

corresponds to a Topological Quantum Field Theory (TQFT). It should be independent of the complex structure chosen. The projectively flat connection gives locally a natural identification between the quantum vector spaces corresponding to different complex structure. Globally the choice reduces to on action of the mapping class group $\Gamma-$ (which is also a topological invariant). Hence, this action gives invariants of the TQFT in question. 


\subsection{The Relation to Berezin-Toeplitz Quantization}

Note in the following that $f \in C^{\infty}\left(\mathcal{M}_{s}\right)$, i.e., f is a smooth function on the moduli space of connections, respectively of bundles.

Proposition 5. (Andersen [1]) For $\sigma_{0}, \sigma_{1} \in \mathcal{T}$, denote by $P_{\sigma_{0}, \sigma_{1}}^{\text {end }}$ the parallel transport from $\sigma_{0}$ to $\sigma_{1}$ in $\operatorname{End}\left(\mathcal{V}_{m}\right)$, then

$$
\left\|P_{\sigma_{0}, \sigma_{1}}^{\mathrm{end}} T_{f, \sigma_{0}}^{(m)}-T_{f, \sigma_{1}}^{(m)}\right\|=O(1 / m) .
$$

To show this Andersen uses Theorem 2 (BMS), the deformation quantization of Theorem 3 and carries out further hard and ingenious work. This proposition implies that the Toeplitz operator are asymptotically covariantly constant.

Proposition 6. (Andersen [1]) Let $\phi \in \Gamma$, such that $\phi \in \operatorname{ker} \rho_{m}$, then

$$
T_{f, \sigma}^{(m)}=P_{\phi(\sigma), \sigma}^{\mathrm{end}} T_{f \circ \phi, \phi(\sigma)}^{(m)} .
$$

Theorem 7. (Andersen [1]) Let $\phi \in \Gamma$, such that $\phi \in \bigcap_{m \in \mathbb{N}} \operatorname{ker} \rho_{m}$, then $\phi$ induces the identity on $\mathcal{M}_{s}$.

Proof: By Proposition 6 and the linearity in the function argument of the Toeplitz operators we have

$$
T_{f-f \circ \phi, \sigma}^{(m)}=T_{f, \sigma}^{(m)}-T_{f \circ \phi, \sigma}^{(m)}=P_{\phi(\sigma), \sigma}^{\mathrm{end}} T_{f \circ \phi, \phi(\sigma)}^{(m)}-T_{f \circ \phi, \sigma}^{(m)} .
$$

We take the norm of this expression and use Proposition 5 to conclude

$$
\left\|T_{f-f \circ \phi, \sigma}^{(m)}\right\|=O(1 / m)
$$

or,

$$
\lim _{m \rightarrow \infty}\left\|T_{f-f \circ \phi, \sigma}^{(m)}\right\|=0 .
$$

This implies $|f-f \circ \phi|_{\infty}=0$ by Theorem 2, Part a, for all $f$, hence $\phi=$ id considered as element acting on the moduli space.

Now Theorem 4 follows from known results which elements of the mapping class group act trivially on the moduli space of connections. 


\section{Appendix: Symplectic Form on $\mathcal{M}$}

Here we consider only the case without holonomy. Let $\mathcal{A}_{F}$ be the affine space of all flat $\mathrm{SU}(n)$ connections, and $\mathfrak{g}=\mathfrak{s u}(n)$. The tangent vectors at $A \in \mathcal{A}_{F}$ can be given as $\alpha, \beta \in \Omega^{1}(X) \otimes \mathfrak{g}$. On this space

$$
\Omega_{A}(\alpha, \beta)=\frac{\mathrm{i}}{2 \pi} \int_{X} \operatorname{Tr}(\alpha \wedge \beta)
$$

is a skew-symmetric form which is invariant under the gauge group and hence descends to $\mathcal{M}=\mathcal{A}_{F} / \mathcal{G}$. If we restrict the situation to the irreducible connections, then the quotient $\mathcal{M}_{s}=\mathcal{A}_{F}^{s} / \mathcal{G}$ is a manifold and $\Omega$ descends to a symplectic form on $\mathcal{M}_{s}$

To define the bundle one uses the Chern-Simons (CS) action. Let $N$ be a threemanifold with boundary $\partial N=X$. For a connection $\tilde{A}$ on $N$

$$
\mathrm{CS}(\tilde{A}):=\frac{1}{4 \pi} \int_{N} \operatorname{Tr}\left(\tilde{A} \wedge d \tilde{A}+\frac{2}{3} \tilde{A} \wedge \tilde{A} \wedge \tilde{A}\right) .
$$

For a connection on $X$ we take any extension $\tilde{A}$ to $N$. Also for a gauge transformation $g \in \mathcal{G}$ we take any extension $\tilde{g}: N \rightarrow G$. Then

$$
\theta(A, g):=\exp \left(\mathrm{i}\left(\operatorname{CS}\left(\tilde{A}^{\tilde{g}}\right)-\operatorname{CS}(\tilde{A})\right)\right.
$$

is a $U(1)$-valued well-defined cocycle (the WZW cocycle). It is used to construct the bundle $\mathcal{L}$ over $\mathcal{M}_{s}$ as quotient

$$
\mathcal{L}:=\left(\mathcal{A}_{F}^{s} \times \mathbb{C}\right) / \sim \rightarrow \mathcal{A}_{F}^{s} / \mathcal{G}=\mathcal{M}_{s}
$$

where $(A, z) \sim\left(A^{g}, \theta(A, g) z\right)$.

The one form $\eta(\alpha)=\frac{1}{4 \pi} \int_{X} \operatorname{Tr}(A \wedge \alpha)$ on $\mathcal{A}_{F}$ induces a unitary connection on $\mathcal{L}$, whose curvature is essentially equal to the symplectic form.

\section{References}

[1] Andersen J., Asymptotic Faithfulness of th Quantum SU(n) Representations of the Mapping Class Group, Ann. Math.163 (2006) 347-368, math.QA/0204084.

[2] Andersen J., Deformation Quantization and Geometric Quantization of Abelian Moduli Spaces, Comm. Math. Phys. 255 (2005) 727-745. 
[3] Andersen J., Asymptotic Faithfulness of the Quantum SU(n) Representations of the Mapping Class Group in the Singular Case, (in preparation)

[4] Bordemann M., Meinrenken, E., and Schlichenmaier, M., Toeplitz Quantization of Kähler Manifolds and $g l(n), n \rightarrow \infty$ Limits, Commun. Math. Phys. 165 (1994) 281-296.

[5] Boutet de Monvel L. and Guillemin, V., The Spectral Theory of Toeplitz Operators. Ann. Math. Studies, Princeton University Press, Princeton, 1981.

[6] Jeffrey L., Flat Connections on Oriented 2-Manifolds, Bull. London Math. Soc. 37 (2005) 1-14.

[7] Masbaum G., Quantum Representations of Mapping Class Groups, Journée annuelle SMF 2003, 19-36.

[8] Karabegov A., Deformation Quantization with Separation of Variables on a Kähler Manifold, Commun. Math. Phys. 180 (1996) 745-755.

[9] Karabegov A., and Schlichenmaier, M., Identification of Berezin-Toeplitz Deformation Quantization, J. reine angew. Math. 540 (2001) 49-76, math.QA/0006063.

[10] Schlichenmaier M., Berezin-Toeplitz Quantization of Compact Kähler Manifolds, In: Quantization, Coherent States and Poisson Structures, Proc. XIV'th Workshop on Geometric Methods in Physics (Białowieża, Poland, 9-15 July 1995), A. Strasburger et.al. Eds., Polish Scientific Publisher PWN, 1998, q-alg/9601016, pp. 101-115.

[11] Schlichenmaier M., Deformation Quantization of Compact Kähler Manifolds by Berezin-Toeplitz Quantization, Conference Moshé Flato 1999 G. Dito and D. Sternheimer, Eds., Kluwer, 2000, math.QA/9910137, Vol. 2, pp. 289-306.

[12] Schlichenmaier M., Berezin-Toeplitz Quantization and Berezin Transform, In Long Time Behaviour of Classical and Quantum Systems, 1999, (S. Graffi, A. Martinez, Eds.), World Scientific, 2001, pp. 271-287, math/0009219.

Martin Schlichenmaier

University of Luxembourg

Campus Limpertsberg

162A, Avenue de la Faiencerie

L-8706 Luxembourg

GRAND-DUCHY OF LUXEMBOURG

E-mail address: martin.schlichenmaier@uni.lu 